\title{
ANALYSIS OF TUNING TIME IN MULTIPLE- SECTION CURRENT-INJECTION TUNABLE LASER DIODES
}

\author{
Efraim Buimovich-Rotem ${ }^{1}$, Dan Sadot $^{1}$ \\ 'Ben Gurion University, POB 653 Beer-Sheva,84105 Israel.E-mail: buimov@ee.bgu.ac.il \\ Sadot@ee.bgu.ac.il
}

\begin{abstract}
A generalized model for calculating the tuning time of multiple-section current-injection laser diodes is presented. The method is applied theoretically and experimentally to the Grating assisted co-directional Coupler with Sampled Reflector (GCSR) laser.
\end{abstract}

\section{INTRODUCTION}

Optical packet switching is considered a key technology in the next generation optical communication networks. Different architectures rely on various photonic components such as tunable lasers, tunable filters, etc. to achieve fast optical routing of data. Fast tunable lasers are used to color packets of data according to their destination. Overhead and latency considerations dictate fast tuning time of the order of 50 nanoseconds for implementing efficient packet switching. [1]

Among the different types of tunable lasers those based on the free carrier plasma effect such as DBR, SG-DBR, GCSR, etc., offer the combination of wide tuning range and fast tuning. Such lasers have been implemented in various system demonstrations [2-4]. The inherent physical limitation on tuning time in these lasers is due to the carrier lifetime in the tuning sections, of the order of 1 nanosecond. Achieving fast tuning times requires fast electronic driving combined with accurate selection of the tuning currents (or operating points).

In this paper we present a general approach for calculation of the tuning time in these lasers based on the properties of the laser and the electronic driving. This model can be used to optimize the laser design and to calculate the inherent physical tuning time limitations (assuming ideal electronic driving). Conversely, 
the expected tuning time can be calculated for a particular electronic driving design and can be compared with its inherent physical limitations. We later apply this model to the GCSR laser and calculate the expected tuning time of a particular fast tunable transmitter. The calculations are verified experimentally.

\section{THEORY}

We are interested in obtaining the temporal frequency evolution $f(t)$ of the laser following a change in the tuning currents designed to switch the laser's wavelength from a certain source wavelength $\lambda_{S}$ to a destination wavelength $\lambda_{D}$. We define the laser's detuning as its frequency deviation relative to the destination wavelength using: $\Delta f(t)=f(t)-f_{D}$, where $f_{D}$ is the laser frequency at the destination wavelength. A relevant problem is to find the tuning time $t_{T X}$ for which $\left|\Delta f\left(t_{T X}\right)\right|=f_{\text {sys }}$, where $f_{s y s}$ is the required frequency accuracy as defined for a system in which the laser is to be used. For practical reasons, the longest tuning time over the entire set of wavelengths defined for the laser will be regarded as the general tuning time.

We define for each of the laser's tuning sections a detuning coefficient $(\partial f / \partial N)_{i}$ where the index $i$ indicates the $\mathrm{i}-t h$ tuning section, as the change in the laser's cavity mode frequency caused by a change in the carrier concentration $N$ at that section. We assume these coefficients are constant and verify that experimentally. A change $\Delta N_{i}$ in the carrier concentration will therefore cause a frequency change $\Delta f=(\partial f / \partial N)_{i} \Delta N_{i}$ provided the tuning is within the limits of continuous tuning and a mode-hop does not occur. While within the continuous tuning limits the detuning coefficient can be written as:

$$
\left(\frac{\partial f}{\partial N}\right)_{i}=\left(\frac{\partial f}{\partial n^{\prime}}\right)_{i} \cdot\left(\frac{\partial n^{\prime}}{\partial N}\right)_{i}
$$

where $n^{\prime}$ is the real part of the refractive index. The first term in the RHS is calculated by solving the amplitude and phase conditions of the cavity [6] and is dependant of the tuning section structure. The second term in the RHS is given by $\partial n^{\prime} / \partial N=\beta_{p l}$, where $\beta_{p l}$ is the free-carrier plasma coefficient [6] and is therefore equal for all the tuning sections.

We define $\Delta N(t)=N(t)-N_{D}$ as the change in the carrier concentration in the tuning section relative to the predefined steady state value $N_{D}$ of the 
destination wavelength $\lambda_{D}$. The carrier concentration $N(t)$ is obtained by solving for each tuning section the rate equation: [6]

$$
\frac{d N}{d t}=\frac{\eta i(t)}{q V_{a}}-\left(A N+B N^{2}+C N^{3}\right)
$$

where $\eta$ is the current confinement factor, $i(t)$ is the tuning current, $V_{a}$ is the junction volume, $q$ is the electron charge, A,B, and $\mathrm{C}$ are the non-radiative, bimolecular and Auger recombination coefficients respectively. The tuning current $i(t)$ can be taken as a theoretical step function in order to investigate the physical limitations of the laser, or as the actual current pulse of the current driver used in a real laser transmitter. The effect of an improved current pulse such as a predistortion pulse can be explored in this manner.

Finally, we assume that within the boundaries of continuous tuning, where only one longitudinal mode exists, the tuning sections affect the laser frequency only through their phases. Therefore, the laser frequency detuning is given by summing the contributions of all individual tuning sections:

$$
\Delta f(t)=\sum_{i}\left(\frac{\partial f}{\partial N}\right)_{i} \Delta N_{i}(t)
$$

Since the required frequency accuracy in practical systems is usually smaller than the entire continuous tuning range, this model is sufficient for the purpose of calculating the tuning time $t_{T X}$.

\section{TUNING TIME OF GCSR LASER}

The GCSR laser consists of four sections: a multiple quantum well gain section, a co-directional coupler, a phase matching section, and a sampled Bragg reflector section. The tuning range is $1529-1561 \mathrm{~nm}$. A more detailed description of the laser structure is available in [5].

The reflection peaks of the sampled reflector are about $4 \mathrm{~nm}$ apart. The coupler acts as a coarse filter, and the current applied to the coupler section is controlled to select one reflector peak. Fine tuning is achieved by tuning the reflector peak to a desired wavelength and adjusting the phase current to align the cavity mode comb to the selected wavelength.

In fig. 1 a partial mode-plane map of the laser output power versus the coupler and reflector currents is presented. The boundaries of a single longitudinal mode are highlighted. The detuning coefficients are defined inside those boundaries and mode hops of $\pm 0.2 \mathrm{~nm}$ and $\pm 4 \mathrm{~nm}$ occur when the mode boundaries are crossed. 


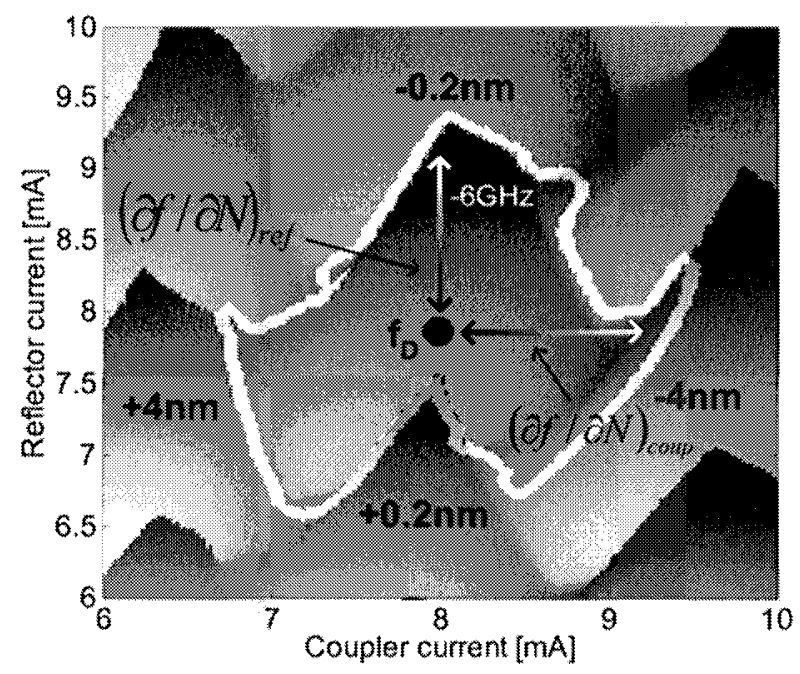

Figure 1. Map of output power versus coupler and reflector sections tuning currents of GCSR laser showing lasing modes.

The detuning coefficients were measured empirically by introducing small increments to the tuning currents and measuring the change in laser frequency. The amount of change in the carrier concentration was then calculated by solving (2) in steady state. The physical parameters of the laser are given in [7]. The detuning coefficients measurement was repeated at different operating wavelengths, and the coefficients were verified to be constant. The measured values of the tuning coefficients $\{\partial f / \partial N\}_{i}$ were $1.64 \cdot 10^{-22}, 3.27 \cdot 10^{-23}, 4.07 \cdot 10^{-22}\left[\mathrm{GHz} \cdot \mathrm{m}^{3}\right]$ for the coupler, phase and reflector sections respectively. The assumption expressed in (3) was also verified experimentally with an accuracy of $10 \%$.

We shall proceed to calculate the longest tuning time expected in a particular GCSR laser transmitter [7].

We define $I_{\max }, I_{\min }$ as the high and low limits of the tuning currents of each section. We shall focus on the worst case where all tuning sections are switched from $I_{\max }$ to $I_{\min }$ [8]. In fig.2 we present an example of the frequency evolution calculation after the reflector current is switched. In fig.2(a) the current pulse $i(t)$ is shown. The current pulses supplied by the transmitter to the tuning sections of the laser were measured using a digital scope and were used to solve (2). The reflector current is switched from $25 \mathrm{~mA}$ to $1 \mathrm{~mA}$, and the current settling time is $\sim 30$ ns. In fig.2(b) the carrier concentration $N(t)$ in the reflector obtained by solving (2) numerically is shown. In fig.2(c) the frequency detuning 
$\Delta f(t)=(\partial f / \partial N) \Delta N(t)$ is presented. The frequency at the center of the mode, as shown in fig. 1 , is taken as the destination frequency $f_{D}$. As also shown in fig.1, beyond the vertical mode boundaries a cavity mode hop of $0.2 \mathrm{~nm}$ occurs, which is associated with changing the reflector current beyond a certain value. While inside the mode boundaries, a frequency detuning of up to $6 \mathrm{GHz}$ can be observed between the mode center and the upper boundary. Consequently, fig.2(c) is valid only for $\Delta f \leq 6 G H z$.

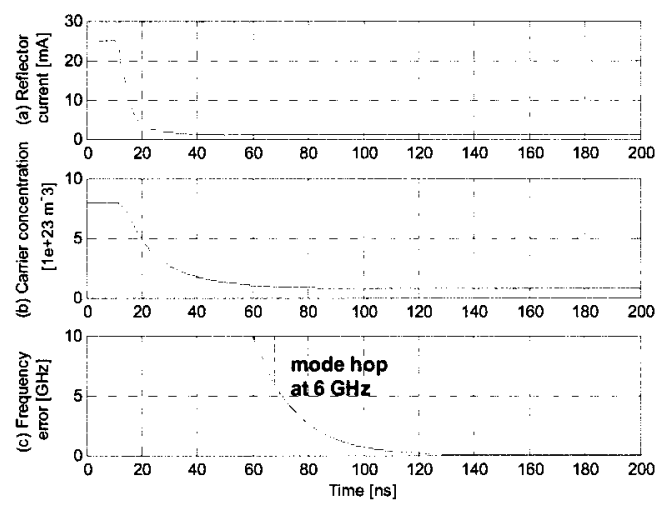

Figure 2. Tuning time calculation. (a) Reflector section current. (b) Reflector section carrier concentration. (c) Laser frequency detuning.

We investigated the sensitivity of the tuning time to $I_{\min }$ and $I_{\max }$ separately. In the first set of calculations we examined the effect of $I_{\max }$ on the tuning time. $I_{\min }$ was constant at $2 \mathrm{~mA}$ in all the calculations, and $I_{\max }$ was gradually increased from $25 \mathrm{~mA}$ to $50 \mathrm{~mA}$. Fig.3(a) presents the individual contributions of each tuning section (while keeping the other two fixed), and the overall tuning time while switching all three tuning currents from $I_{\max }$ to $I_{\min }$.

In the second set of calculations we examined the effect of $I_{\min }$ on the tuning time. $I_{\max }$ was constant at $25 \mathrm{~mA}$, and $I_{\min }$ was gradually increased from $1 \mathrm{~mA}$ to $15 \mathrm{~mA}$. Fig.3(b) presents the individual contributions of each tuning section assuming switching of only one section, and the overall tuning time assuming switching of all tuning sections from $I_{\max }$ to $I_{\min }$. The required frequency accuracy $f_{\text {sys }}$ was $6 \mathrm{GHz}$ in all cases.

We observe in Fig. 3 that $I_{\min }$ has a strong influence on the tuning time while $I_{\max }$ has very little influence. Further we notice that the reflector section dominates the tuning time, due to its large tuning coefficient, and its large volume, 
which increases the denominator in (2) and slows the tuning process. The dominant effect of the reflector section was also reported previously [8].

We therefore concluded that in a GCSR laser transmitter, the worst case tuning time is primarily a function of the reflector minimal current.

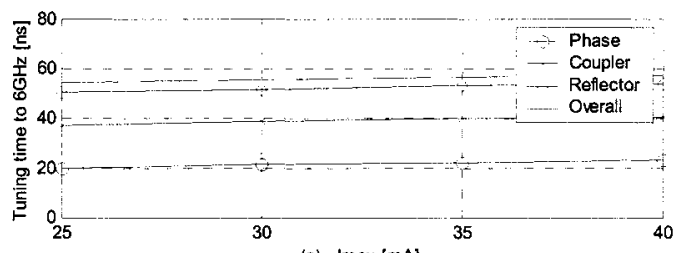

(a) $\operatorname{Imax}[\mathrm{mA}]$

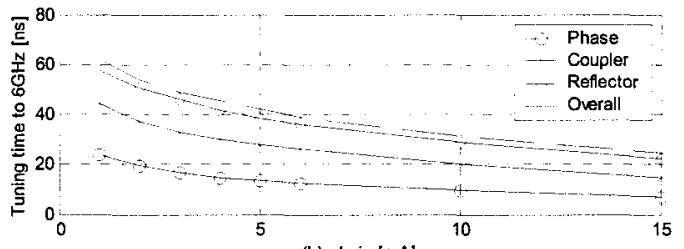

(b) Imin $[\mathrm{mA}]$

Figure 3. Theoretical calculations of tuning time with $6 \mathrm{GHz}$ accuracy. (a) As a function of the maximal current $I_{\max }$. (b) As a function of the minimal current $I_{\min }$.

\section{Experimental verification}

In this section we present the results of a set of experiments that was performed in order to verify the theoretical calculations of tuning time under the worst case conditions described above.

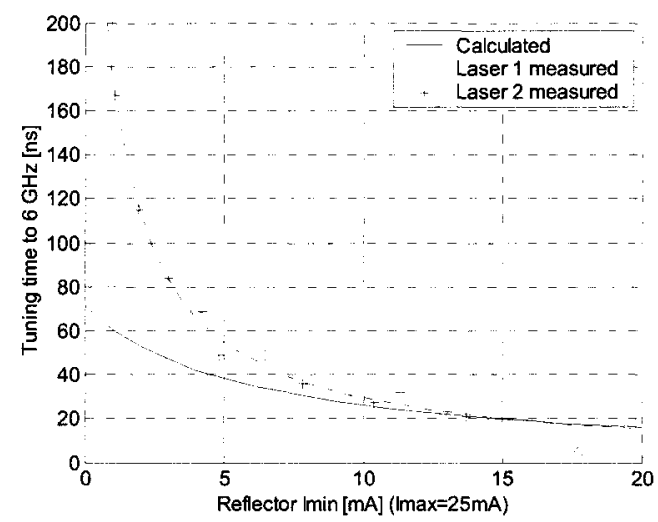

Figure 4. Theoretical and measured tuning time as a function of the reflector current minimal value $I_{\min }$

As noted earlier the frequency at the mode upper boundary was measured to be 
approximately $6 \mathrm{GHz}$ less than the frequency at the mode center, throughout the laser tuning range. By using a $20 \mathrm{GHz}$ FWHM band-pass optical filter tuned to the frequency at the center of the destination mode, the mode hop into the destination mode, and consequently into filter pass band, produces a sharp increase in the detected signal, indicating the instant when the laser frequency obeys $\Delta f(t)=6 \mathrm{GHz}$.

In Fig. 4 we present the calculated and measured tuning time for two GCSR lasers. 15 operating points were selected with reflector currents ranging between $1 \mathrm{~mA}$ and $18 \mathrm{~mA}$. For currents above $7 \mathrm{~mA}$ there is a very good agreement between measured and calculated results.

When switching to operating points with reflector currents below $7 \mathrm{~mA}$ the measured tuning times are increasingly longer than the calculated values. In order to achieve a tuning time of less than $50 \mathrm{~ns}$ with a frequency accuracy of $6 \mathrm{GHz}$ the reflector current of the working points should be above $7 \mathrm{~mA}$.

In order to investigate the long tuning times observed while switching to low currents we measured the voltage across the reflector during the tuning process. The reflector voltage is a direct indicator of the carrier concentration [6] thus providing an important view into the laser dynamics.

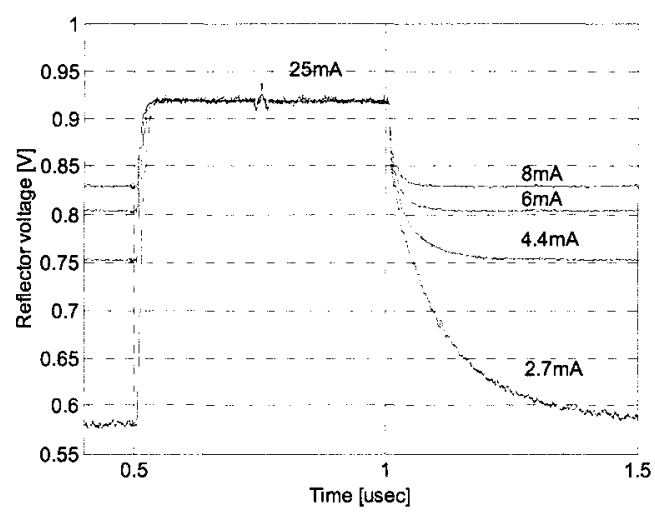

Figure 5. Reflector voltage during switching between $25 \mathrm{~mA}$ and lower currents.

As shown in Fig.5, there is a strong correlation between the measured tuning times and the measured reflector voltage transients. As the final reflector current decreases the voltage settling time increases considerably.

Furthermore, the voltage-current curve of the reflector section was measured and its knee point was observed at $5 \mathrm{~mA}$. This indicates that most of the mismatch between the theoretical and measured tuning times occurs while the reflector current is below the diode cut-off current. We conclude that while the tuning section operates at low forward bias (2) does not provide an accurate model for the tuning section dynamics and a better model is required. 


\section{CONCLUSIONS}

We have presented a method for calculating the tuning time of multiple section current injection lasers. This method uses a system engineering approach that incorporates the electronic drive circuits together with basic semiconductor dynamics theory and the optical properties of the tuning sections which can be either calculated or easily measured.

The method was applied to the GCSR laser, and the limitations on tuning time in this laser were calculated theoretically and verified experimentally.

The theoretical calculation of tuning time which is based on the carrier concentration rate equation has proved to be accurate for currents above $7 \mathrm{~mA}$. Below this current we observed longer tuning times, which were also accompanied by a slow change in the reflector voltage. This indicates that at small forward bias a different dynamic model has to be considered.

\section{REFERENCES}

[1] D. Sadot, and I. Elhanany, "Optical switching speed requirements for Terabit/sec packet over WDM networks," IEEE Photonics Technology letters, 12, no., 4, pp. 440442, April 2000

[2] S. Rubin, E. Buimovich, G. Ingber, and D. Sadot, "Implementation of an ultra-fast widely-tunable burst-mode $10 \mathrm{Gbps}$ transcevier," IEE Electronic Letters, vol. 38, no. 23, pp.1462-1463, Nov. 2002.

[3] P. J. Rigole, M. Shell, S. Nilsson , D. J. Blumental and E. Berglind, "Fast wavelength switching in a widely tunable GCSR laser using pulse predistortion technique," in Proc. of OFC conf., Dallas, TX, USA, 1997, vol. 6, paper WL63, pp231-232.

[4] Y. Fukashiro, K. Shrikhande, M. Avenarius, M. S. Rogge, I. M. White, D. Wonglumsom, L. G. Kazovsky, "Fast and fine wavelength tuning of a GCSR laser using a digitally controlled driver, " in Proc. of OFC conf., Baltimore, MD, USA, 2000, vol.2, pp.338 -340 .

[5] P. J. Rigole, S. Nilsson, L. Backbom, B. Stalnacke, E. Berglind, E. J. P. Weber, B. Stoltz, "Quasi-continuous tuning range from 1520 to $1560 \mathrm{~nm}$ in a GCSR laser, with high power and low tuning currents," IEE Electronics Letters, vol. 32, no.25, pp. 23522354, Dec. 1996.

[6] M.C.Amann J.Buus, "Tunable Laser Diodes", Artech House pub. 1998.

[7] E. Buimovich, D. Sadot, "Physical limitations of tuning time and system considerations in implementing fast tuning of GCSR lasers", Journal of Lightwave Technology, 22, no.2, pp. 582-588, February 2004.

[8] J. E. Simsarian, A. Bharwaj, K. Dreyer, J. Gripp, O. Laznicka, K. Sherman, Y. Su, C. Webb, L. Zhang, M. Zirngibl, "A widely tunable laser transmitter with fast accurate switching between all channel combinations," in Proc. of ECOC conf., Copenhagen, Denmark, 2002, paper 3.3.6. 